\title{
Ultrasound-Guided Cytosolic Protein Delivery via Transient Fluorous Masks
}

Janna N. Sloand ${ }^{\dagger}$, Theodore T. Nguyen $\ddagger$, Scott A. Zinck§, Erik C. Cook", Tawanda J. Zimudzi”, Scott A. Showalter", Adam B. Glick ${ }^{\ddagger}$, Julianna C. Simon§, and Scott H. Medinat,

†Department of Biomedical Engineering, The Pennsylvania State University, University Park, PA 16802

‡Department of Veterinary and Biomedical Sciences, The Pennsylvania State University, University Park, PA 16802

§Graduate Program in Acoustics, The Pennsylvania State University, University Park, PA 16802

"Department of Chemistry, The Pennsylvania State University, University Park, PA 16802

\#Materials Research Institute, The Pennsylvania State University, University Park, PA 16802

Content

Page Number

Table S1. List and chemical structure of screened FTags $\quad$ S1

Figure S1. GFP dispersion screen using 0.01. 0.1, and $1 \mathrm{mM} \mathrm{FTag} \quad$ S2

Figure S2. Macroscopic images of GFP dispersion in PFH S3

Figure S3: ${ }^{19} \mathrm{~F}-\mathrm{NMR}$ chemical shifts and integration $\quad$ S4

Figure S4. Overlay of PFNA and GFP-PFNA ${ }^{19}$ F-NMR spectra $\quad$ S5

Figure S5. Lorentzian-Gaussian peak fitting of ${ }^{19}$ F-NMR data $\quad S 6$

$\begin{array}{ll}\text { Figure S6. Fluorescent micrograph of } \mathrm{NE}_{\mathrm{GFP}} \text { particles } & \mathbf{S 7}\end{array}$

Figure S7. Fluorescent micrographs of $\mathrm{NE}_{\mathrm{GFP}}$ and GFP treated A549 cells $\quad$ S8

Figure S8. GFP fluorescence after ultrasound exposure $\quad$ S9

Figure S9. IgG dispersion screen using 0.01, 0.1, and 1 mM FTag $\quad$ S10

Figure S10. Effect of PFNA/PFcyclo:IgG molar ratio on dispersion $\quad$ S11

Figure S11. CD spectra of PFNA- or PFcyclo- masked IgG S12

Figure S12. Particle size of IgG-loaded $\mathrm{NE}_{\mathrm{IgG}}$ nanoemulsions $\quad$ S13

Figure S13. Fluorescent micrographs of $\mathrm{NE}_{\beta \text {-tubulin-Ab }}$ treated $\mathrm{A} 549$ cells $\quad \mathbf{S 1 4}$

Figure S14. Fluorescent micrographs of PFcyclo $\mathrm{NE}_{\mathrm{IgG}}$ treated tumor sections $\quad \mathbf{S 1 5}$

Figure S15. Peptide RP-HPLC and ESI-MS spectra $\quad$ S16

Figure S16. Acoustic pressure of $5 \mathrm{MHz}$ transducer $\quad$ S17

Movie S1. B-mode ultrasound guidance of $\mathrm{NE}_{\mathrm{GFP}}$ nanoemulsions

Movie S2. Doppler ultrasound imaging of $\mathrm{NE}_{\mathrm{GFP}}$ nanoemulsion cavitation 


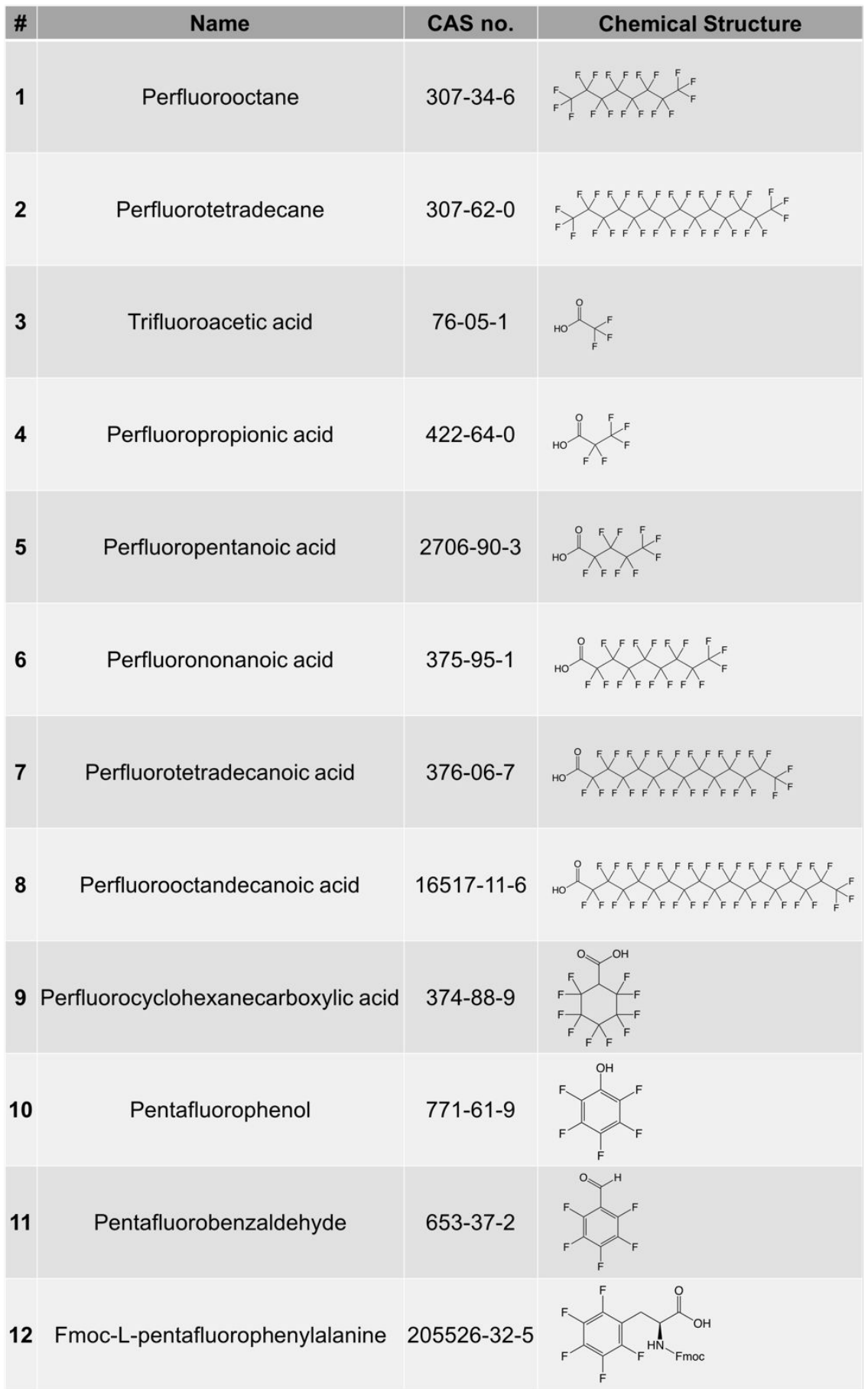

Table S1. List of twelve FTags screened for protein fluorous masking. 


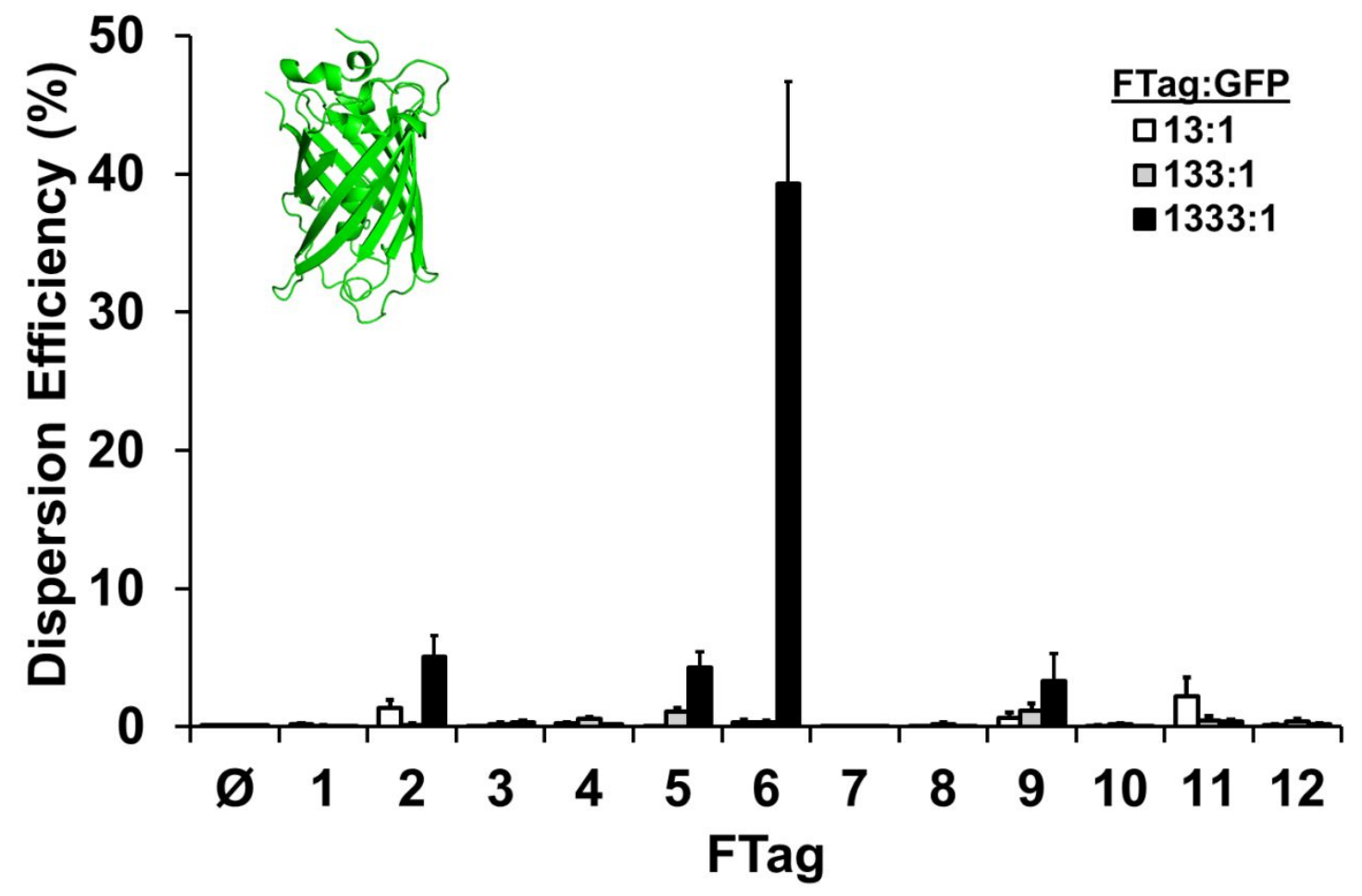

Figure S1. GFP dispersion screen to identify FTags (at a $13-1333: 1$ molar ratio) capable of dispersing GFP into PFH ( $\varnothing=$ no FTag). Data shown as mean \pm SEM for $n=9$ replicates per condition. 
-PFNA

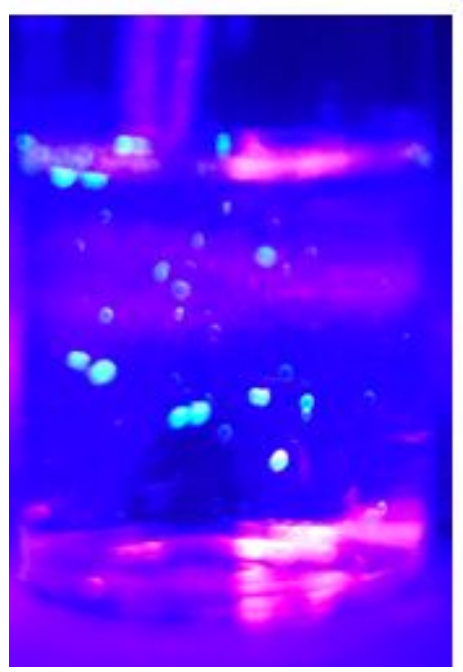

+PFNA

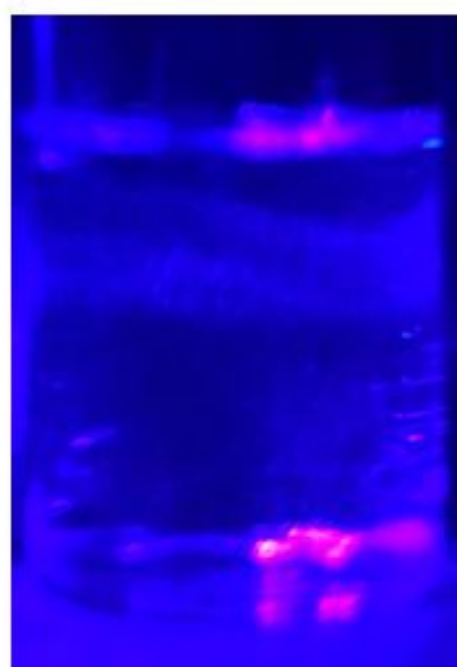

Figure S2. Macroscopic UV images of GFP dispersion in PFH. Without the aid of the FTag (PFNA), GFP is insoluble in PFH and forms large fluorescent precipitates. Addition of the FTag (+PFNA) masks the protein to enable its complete dispersion into the fluorous solvent. 
a

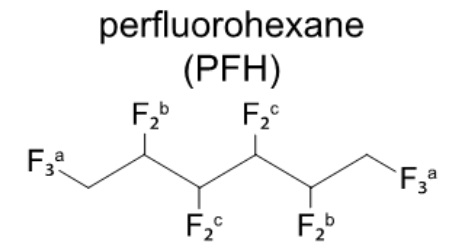

b

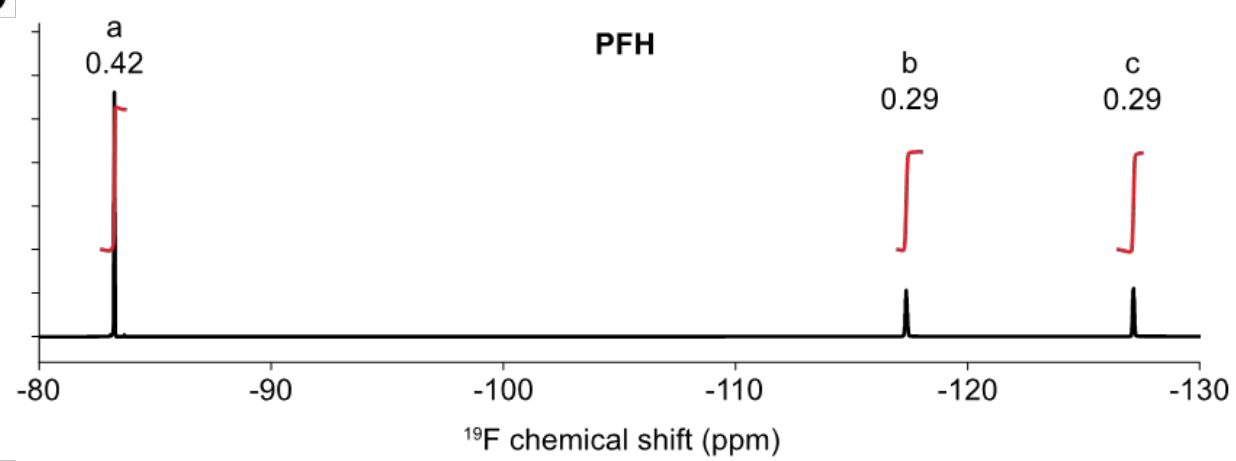

perfluorononanoic acid

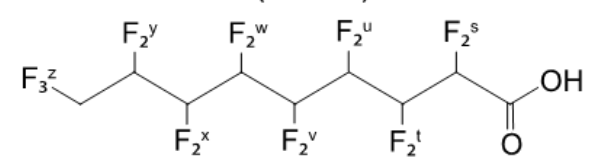

C

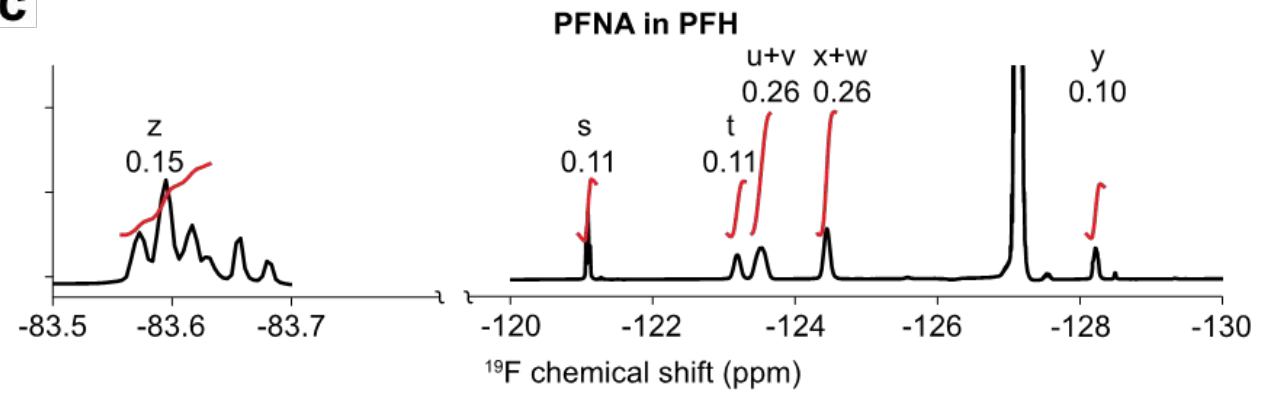

d

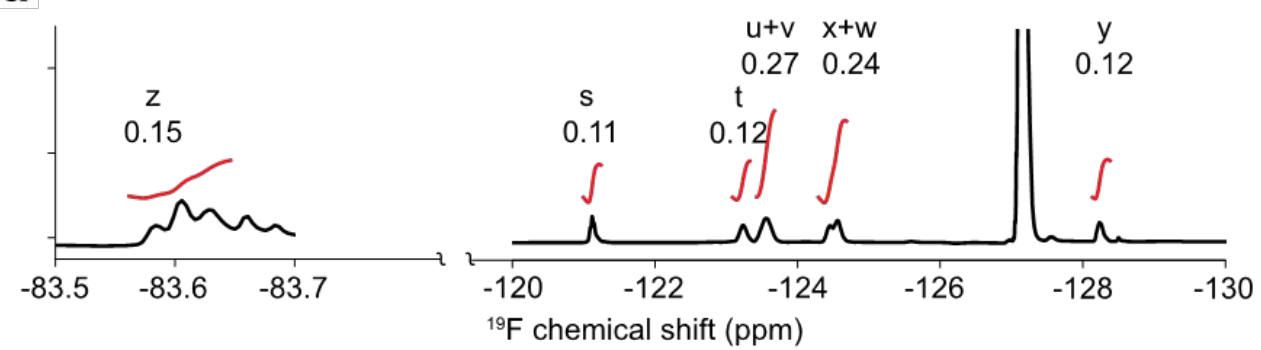

Figure S3. ${ }^{19} \mathrm{~F}-\mathrm{NMR}$ spectra and integration of fluorous compounds. (a) Peak assignments for the PFH solvent and PFNA FTag. ${ }^{19} \mathrm{~F}-N M R$ chemical shifts for (b) PFH solvent, (c) PFNA FTag in $\mathrm{PFH}$, and (d) PFNA masked GFP in PFH. 

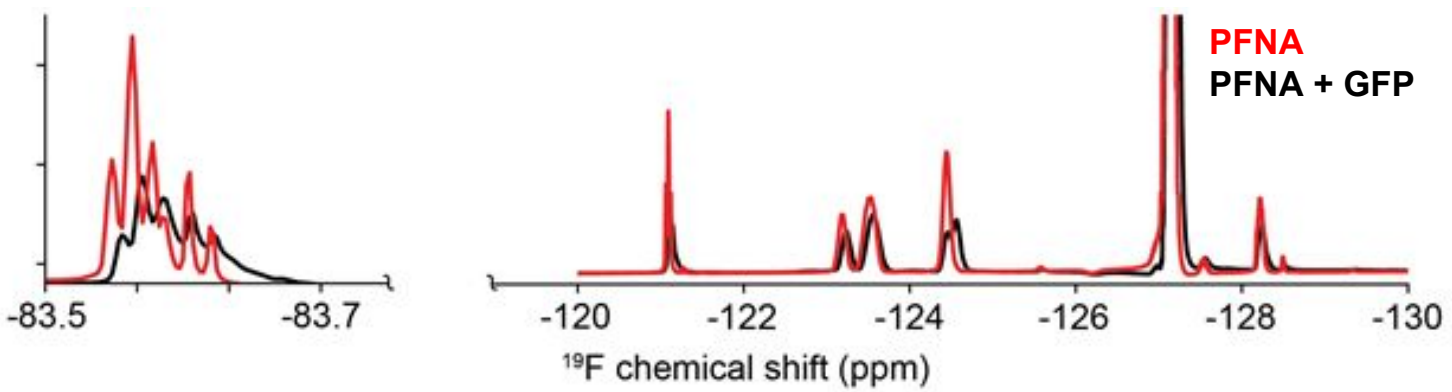

Figure S4. Overlay of PFNA (red) and PFNA-masked GFP (black) ${ }^{19} \mathrm{~F}-\mathrm{NMR}$ spectra in PFH. 


\section{- GFP + PFNA \\ — peak fitting}

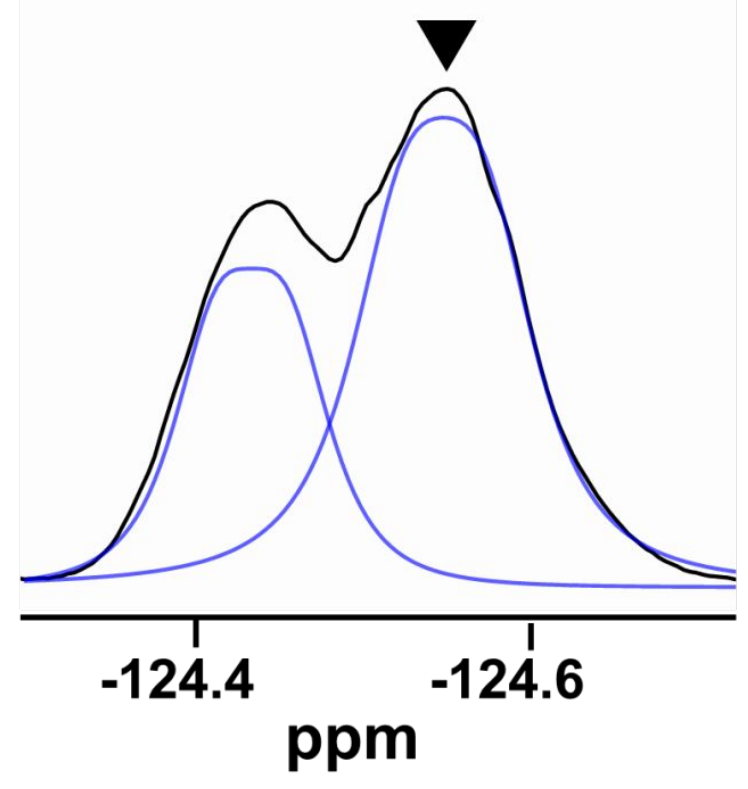

Figure S5. Representative Lorentzian-Gaussian peak fitting results used to calculate molar ratio of bound $(\boldsymbol{\nabla})$ to unbound PFNA with dispersed GFP. 


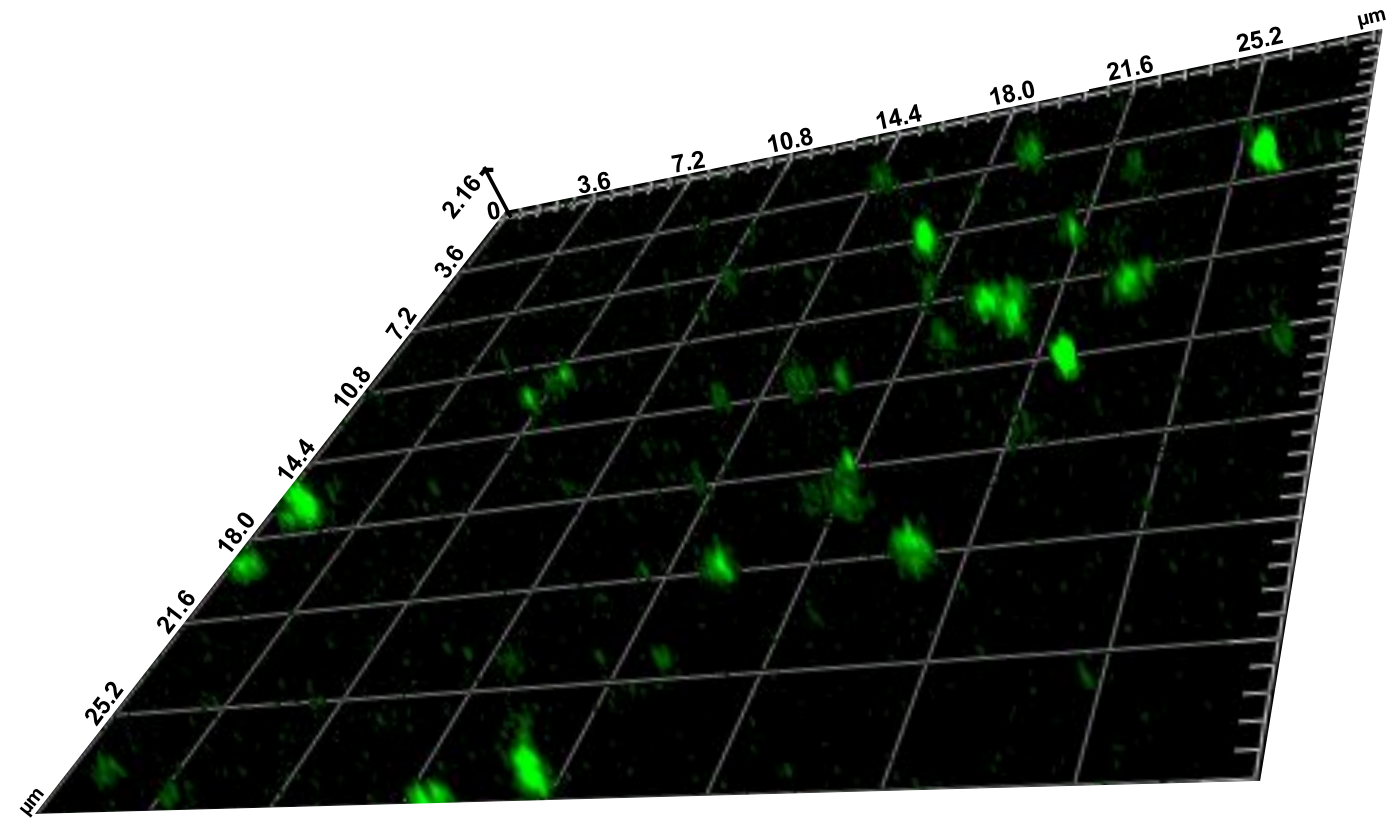

Figure S6. 3D z-stack fluorescent micrograph of $\mathrm{NE}_{\mathrm{GFP}}$ particles. 

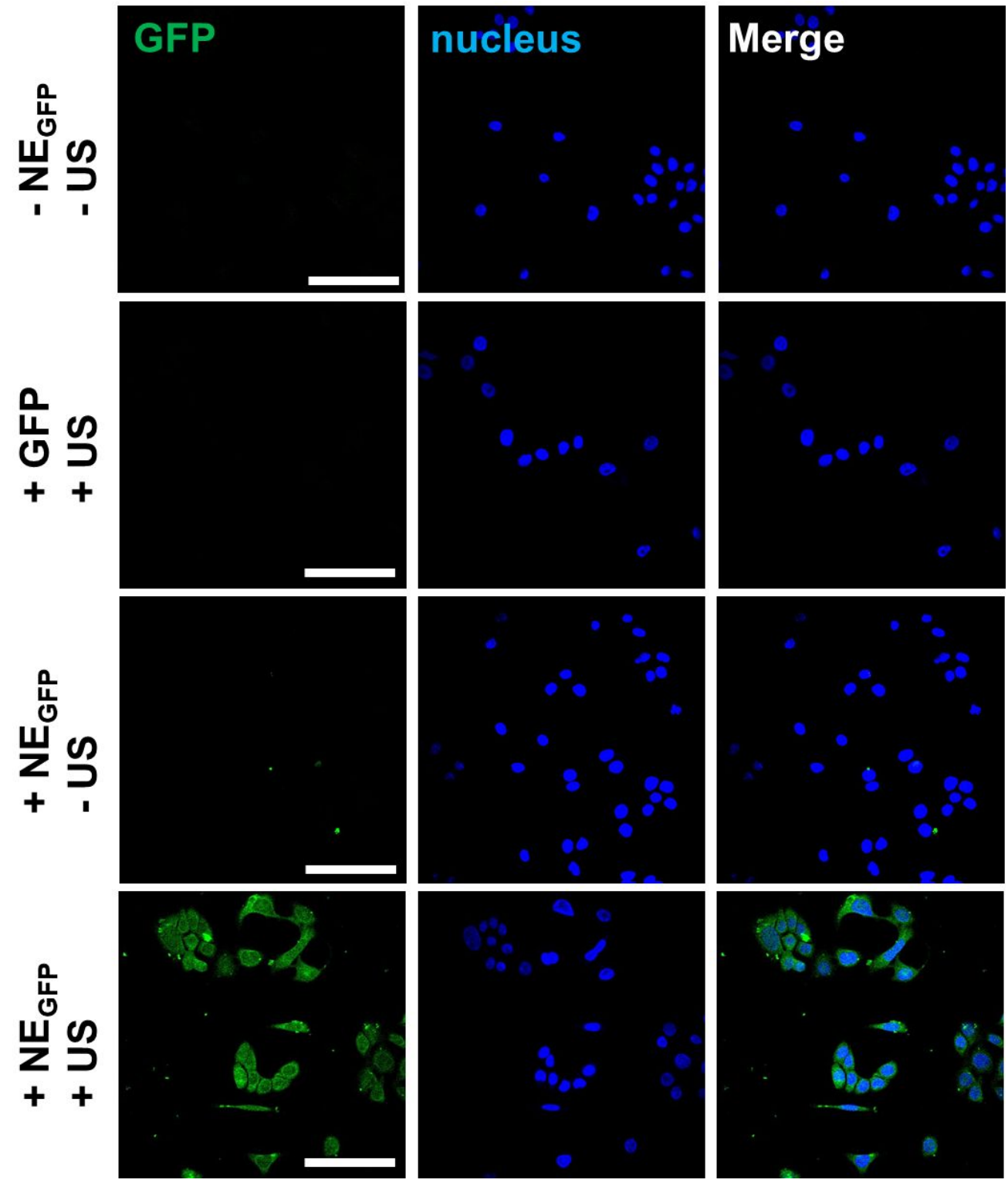

Figure S7. Individual confocal micrographs of untreated A549 cells (-NE $\mathrm{GFP},-\mathrm{US})$, or samples treated with free GFP or $\mathrm{NE}_{\mathrm{GFP}}$ in the absence (-US) or presence (+US) of the acoustic trigger (2 $\mathrm{W} / \mathrm{cm}^{2}$ ). Scale bar $=100 \mu \mathrm{m}$. 


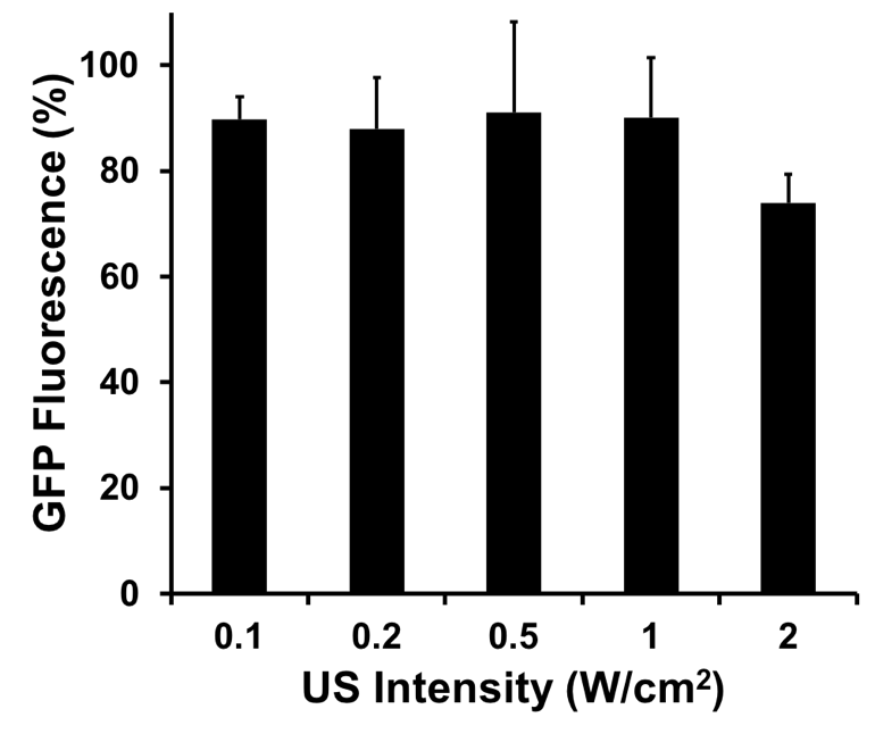

Figure S8. Percentage GFP fluorescence following protein exposure to various ultrasound intensities (90 sec, $20 \%$ duty cycle). 


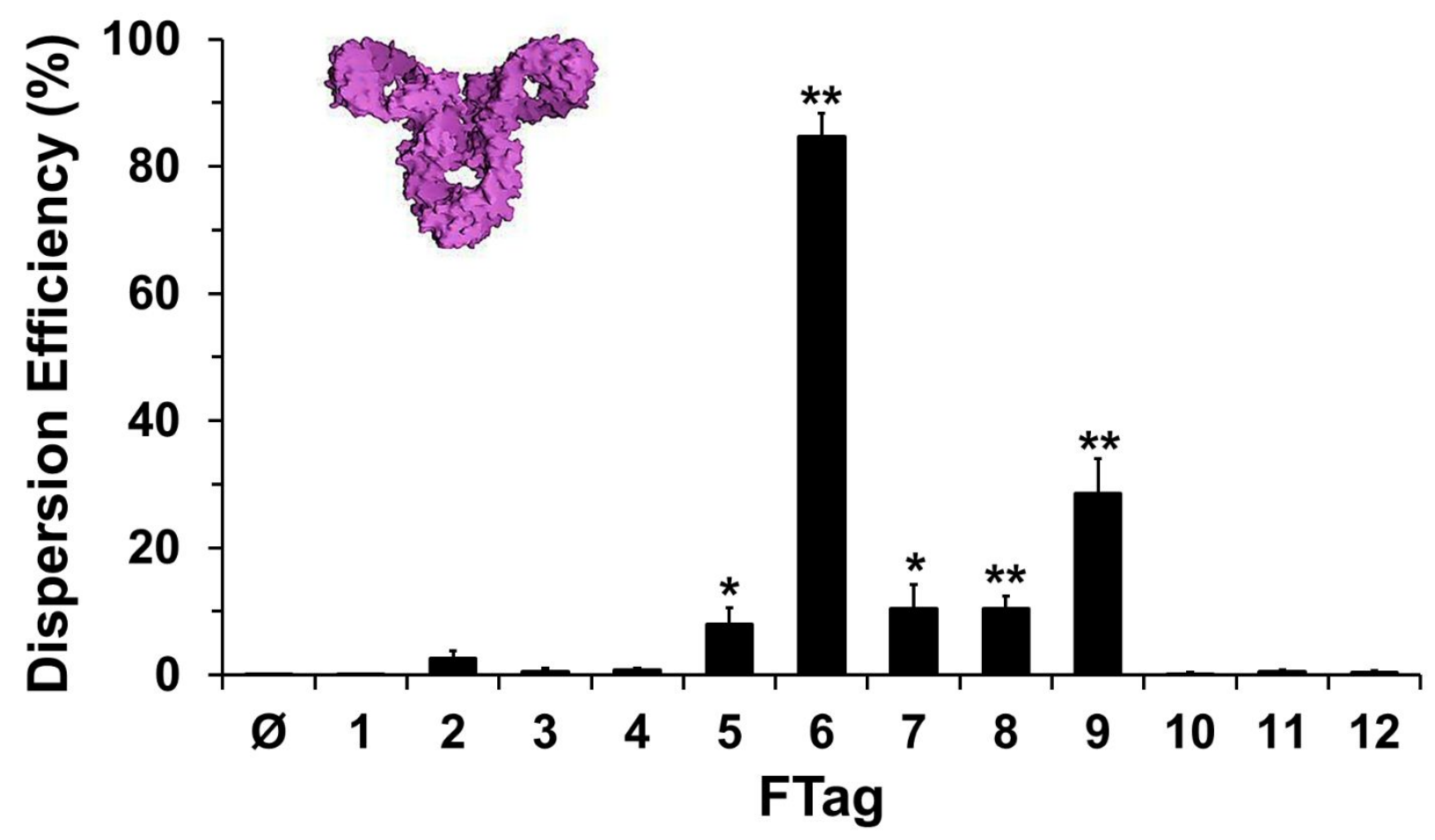

Figure 59. IgG dispersion screen to identify FTags (1000:1 molar ratio) capable of dispersing purified rabbit IgG into PFH $(\varnothing=$ no FTags). Data shown as mean \pm SEM for $n=9$ replicates per condition ( ${ }^{*}$ indicates $p<0.01$, and ${ }^{* *}$ indicates $p<0.001$ relative to $\varnothing$ ). 

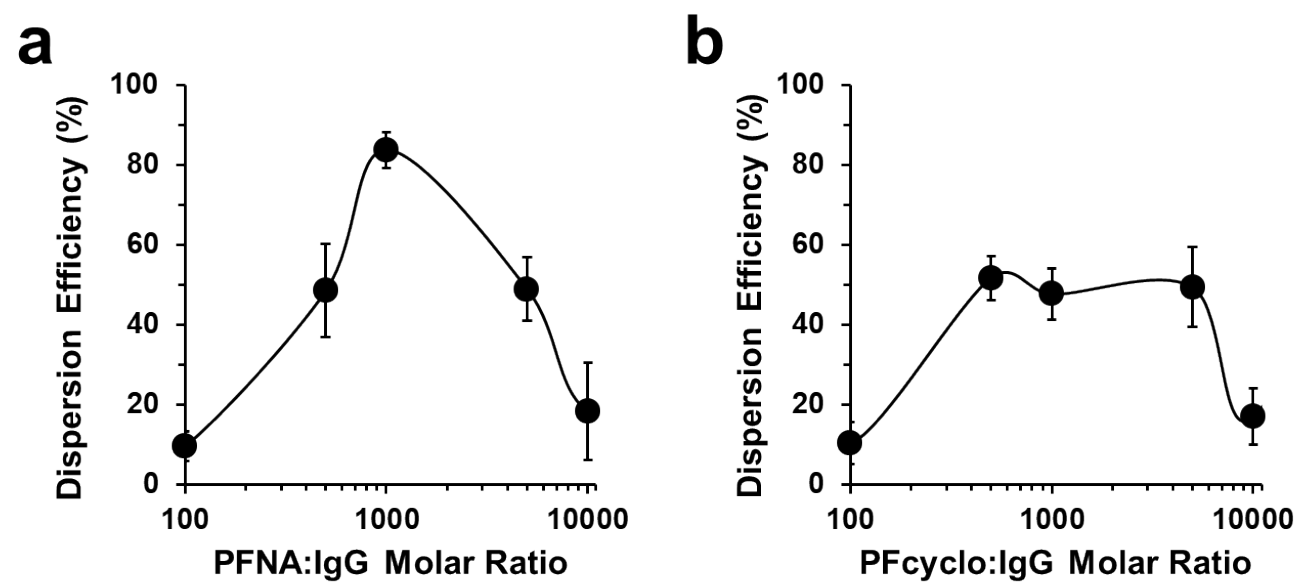

Figure S10. Effect of (a) PFNA and (b) PFcyclo molar ratio on IgG dispersion efficiency in PFH. 


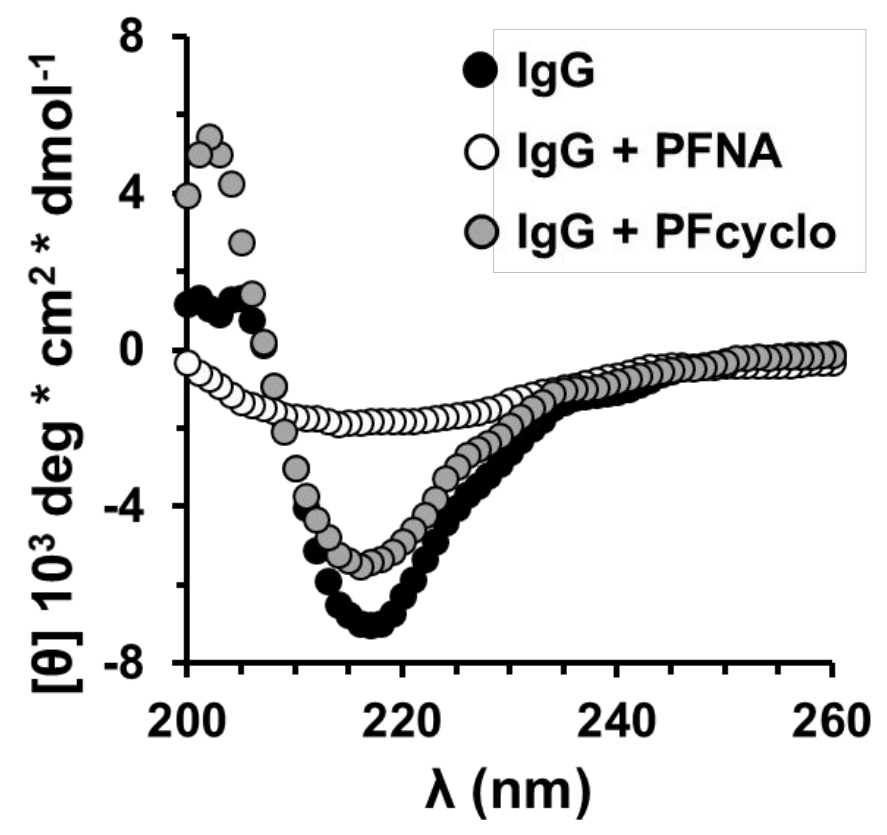

Figure S11. Circular dichroism spectra of IgG before $(\bullet)$ and after complexation with PFNA (०) or PFcyclo (॰) fluorous masking agents (1000:1 molar ratio to lgG). 


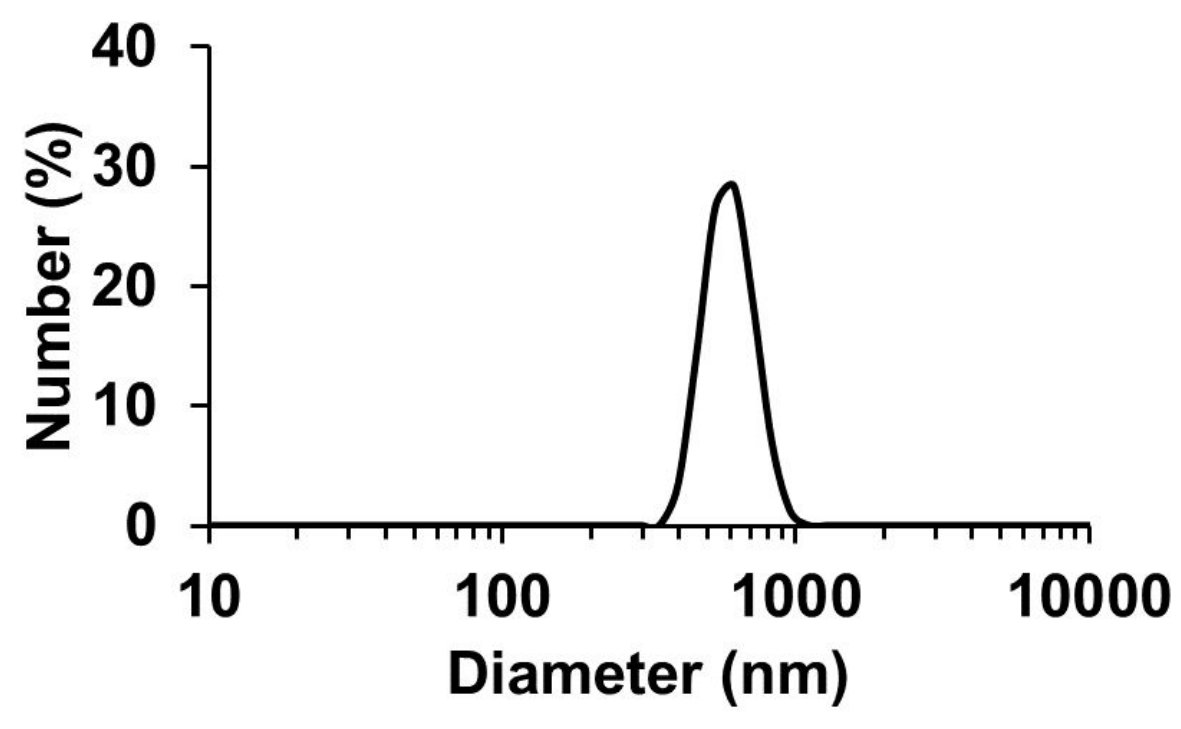

Figure S12. Particle size of IgG-loaded $\mathrm{NE}_{\mathrm{IgG}}$ nanoemulsions, as determined by dynamic light scattering analysis. 


\section{$\mathrm{NE}_{\beta \text {-tubulin }}$}
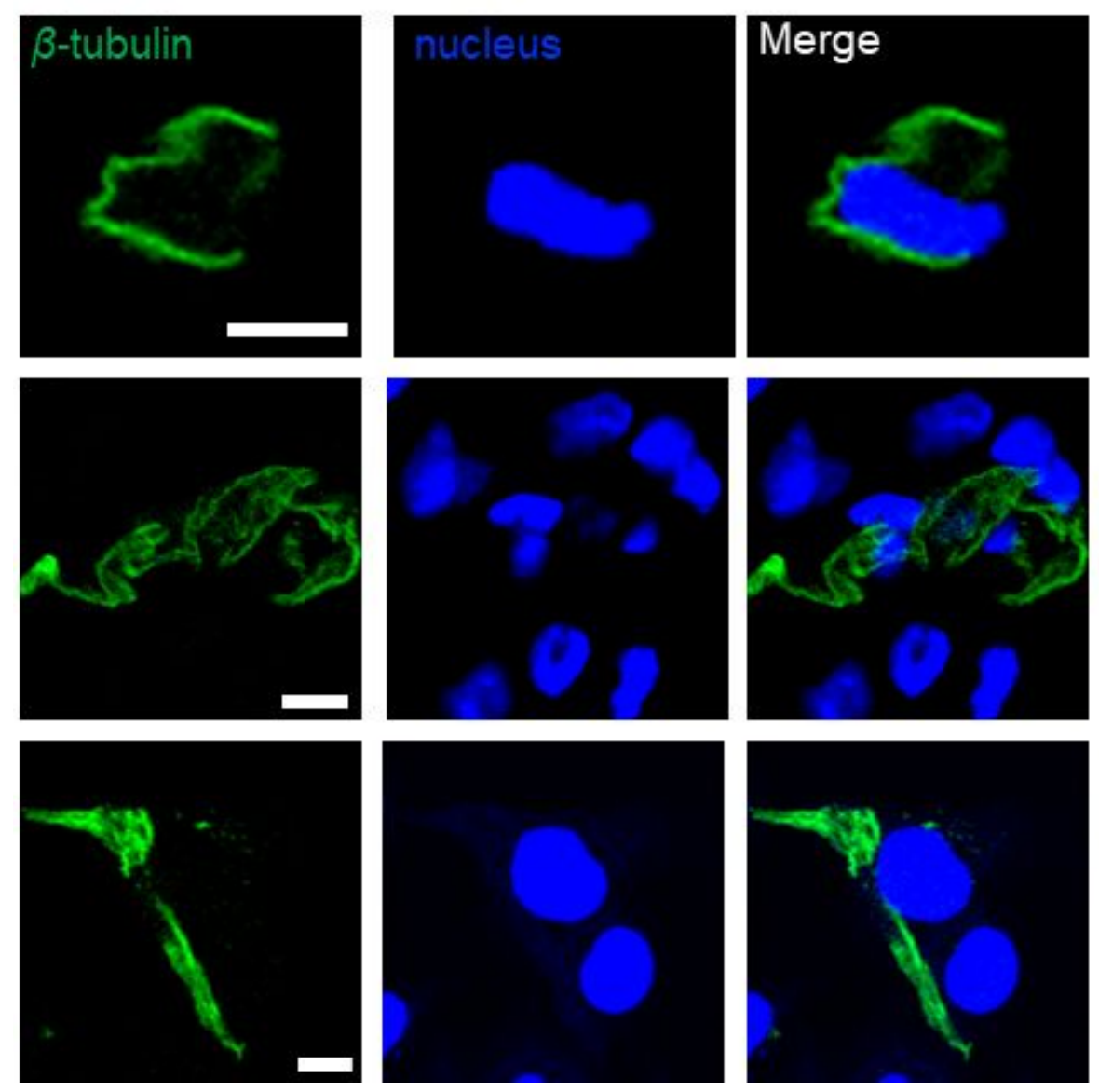
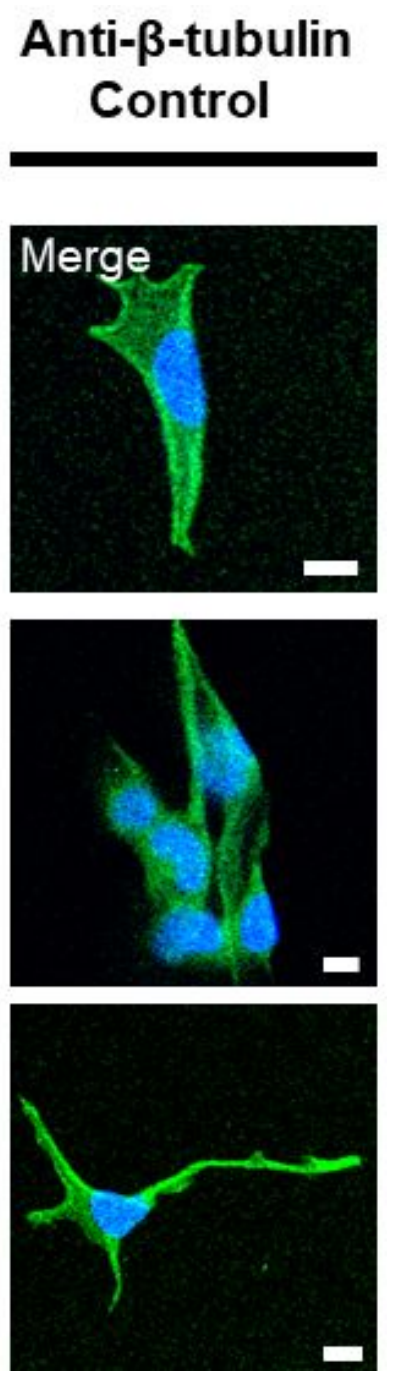

Figure S13. Left: Confocal micrographs of A549 cells following intracellular delivery of FITClabeled anti $\beta$-tubulin antibodies from $\mathrm{NE}_{\beta \text {-tubulin }}$ particles activated with $U S$ at $1.0 \mathrm{~W} / \mathrm{cm}^{2}$ (scale bars $=10 \mu \mathrm{m})$. Three representative view fields are shown. Right: Representative images of A549 cells stained with the free $\beta$-tubulin antibody as a labeling control. 

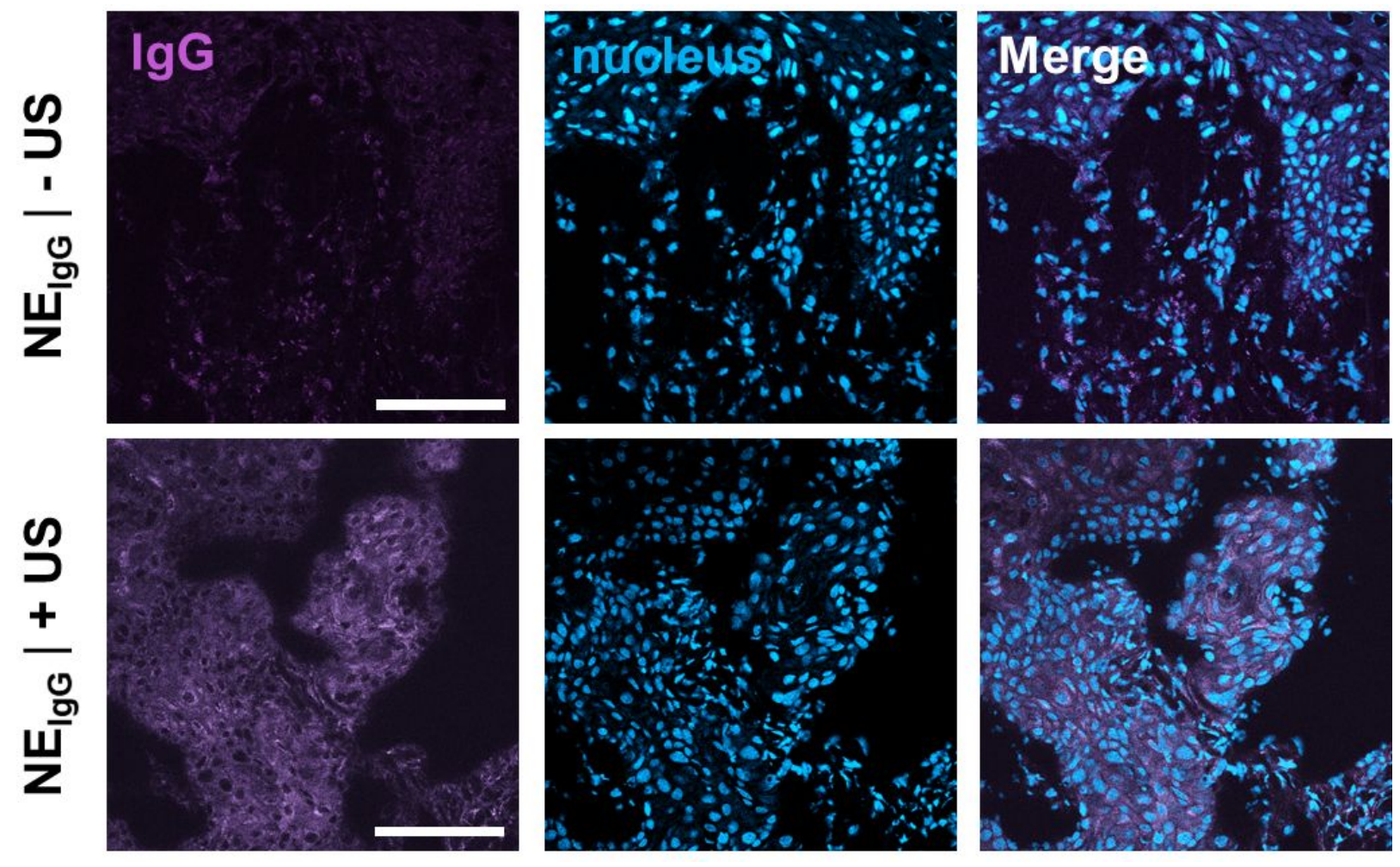

Figure S14. Individual confocal micrographs of sections isolated from InvtTA $x$ tetOHRas ${ }^{G 12 V}$ bitransgenic mice 24 hours after retro-orbital administration of $\mathrm{NE}_{\mathrm{lgG}}$ particles prepared using the PFcyclo FTag (scale bar $=100 \mu \mathrm{m})$. Tumors were either un-insonated (-US) or exposed to multiple pulses of US (+US; $\left.3 \mathrm{~W} / \mathrm{cm}^{2}\right)$ at specific time points. 

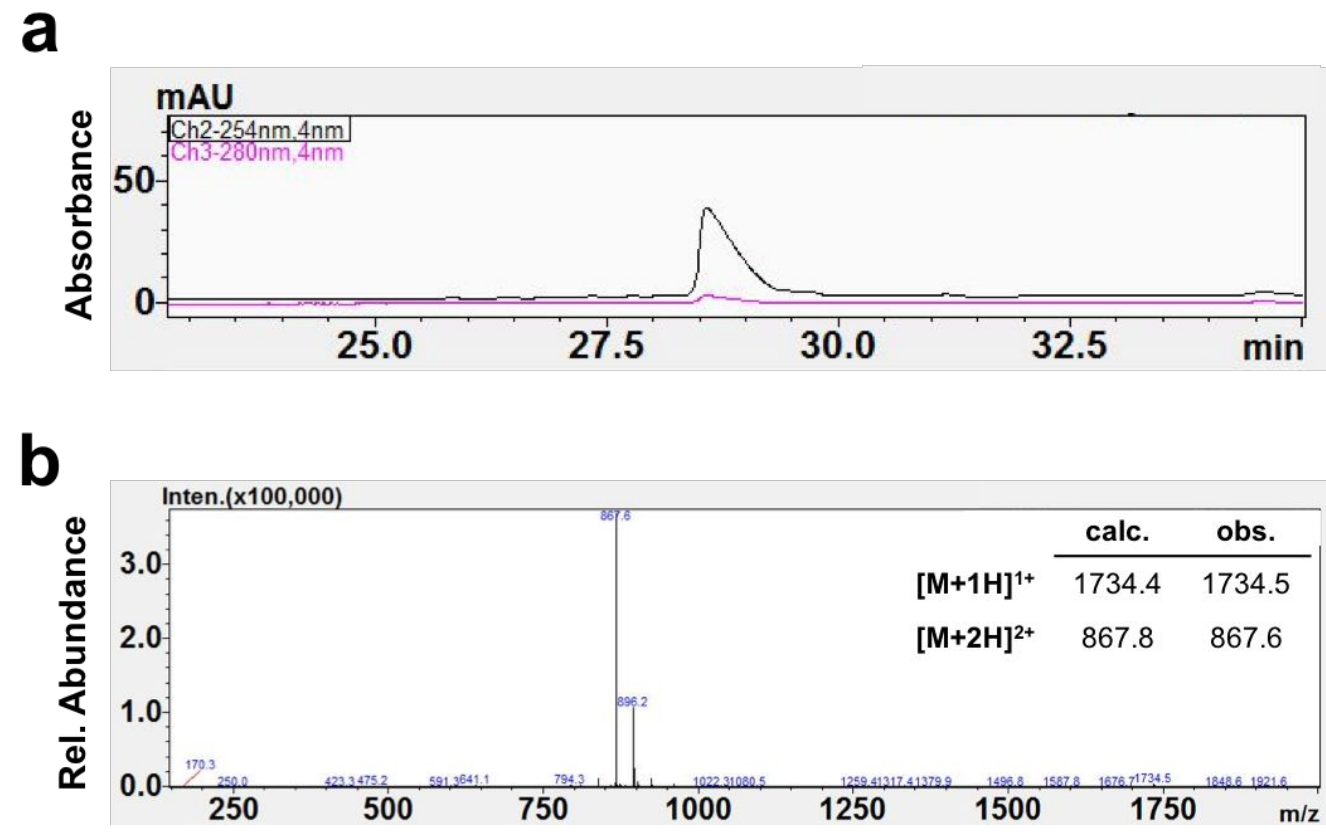

Figure S15. (a) Analytical HPLC (Phenomenex C18; 0\% - 100\% B over $100 \mathrm{~min}$. at $40^{\circ} \mathrm{C}$ ) and (b) ESI (+) mass spectrum of purified RGD peptide (FFFGGGCCGGKGRGD-NH ${ }_{2}$ ). 


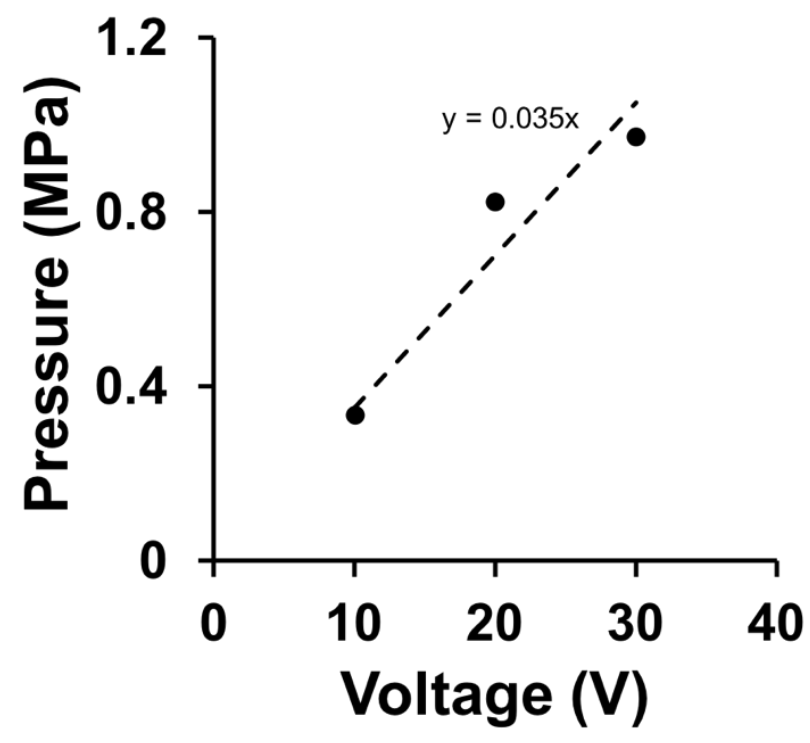

Figure S16. Relationship of acoustic pressure and applied voltage from a $5 \mathrm{MHz}$ ultrasound transducer. 